\title{
Effect of microjet injection on supersonic jet noise
}

\author{
K.B.M.Q. Zaman ${ }^{1}$ and G. G. Podboy ${ }^{2}$ \\ NASA Glenn Research Center \\ Cleveland, $\mathrm{OH} 44135$
}

\begin{abstract}
The effect of microjet ( $\mu$ jet) injection on the noise from supersonic jets is investigated. Three convergent-divergent (C-D) nozzles and one convergent nozzle, all having the same exit diameters, are used in the study. The $\mu$ jets are injected perpendicular to the primary jet close to the nozzle lip from six equally-spaced ports having a $\mu$ jet-to-primary-jet diameter ratio of 0.0054 . Effects in the overexpanded, fully expanded as well as underexpanded flow regimes are explored. Relative to the effect on subsonic jets, larger reductions in the overall sound pressure level (OASPL) are achieved in most supersonic conditions. The largest reductions are typically associated with suppression of screech and transonic tones. For a shock-free, fully expanded case, the OASPL reductions achieved are comparable to that in the subsonic case; the same correlation, found for subsonic jet noise reduction at shallow observation angle, applies.
\end{abstract}

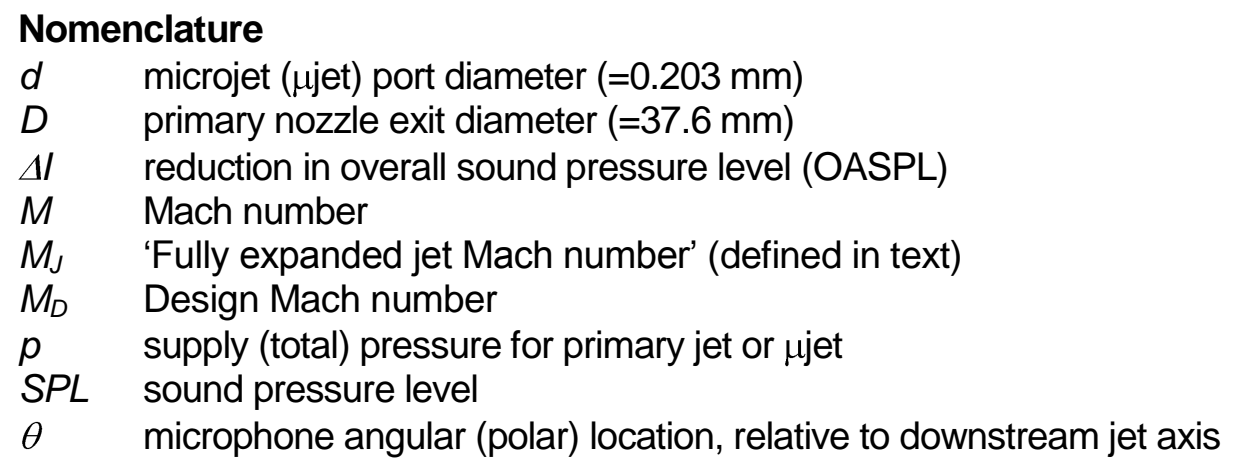

Subscripts

a ambient condition

j primary jet condition

0 stagnation condition

$\mu \quad$ microjet condition

\footnotetext{
${ }^{1}$ Inlet \& Nozzle Branch, Aeropropulsion Division, AIAA Associate Fellow.

${ }^{2}$ Acoustics Branch, Aeropropulsion Division, member AIAA.
} 


\section{Introduction}

Effects of $\mu$ jet injection on the radiated noise from a primary jet have been addressed in many previous studies (e.g., [1-4]). The present study is a continuation of the work reported in [4] in which effects on subsonic jets from a convergent nozzle were addressed. A small but clear noise reduction in the direction of peak noise radiation (i.e., at shallow angles relative to the downstream jet axis) was observed. The reduction in overall sound pressure level (OASPL) improved with increasing $\mu$ jet pressure. It was found that smaller diameter ports with higher driving pressure, but involving less mass fraction and thrust, could produce better noise reduction. As much as $2 \mathrm{~dB}$ reduction in OASPL was achieved with as little as $0.05 \%$ mass fraction. The OASPL reduction in the shallow angle direction correlated with the ratio of $\mu$ jet-to-primary-jet driving pressures normalized by the ratio of the corresponding diameters $\left(p_{\mu} d / p_{j} D\right)$. That is, when all data, including water and $\mathrm{CO}_{2}$ injection data, were plotted as a function of this parameter they collapsed reasonably well. The OASPL reduction $(\Delta l$, in $\mathrm{dB})$ in such a plot increased almost linearly and followed the correlation, $\Delta l=12.0 p_{\mu} d / p_{j} D$. The scaling with $p_{\mu} d / p_{j} D$ was observed only in the jet's downstream direction and thus applied to turbulent mixing noise attenuation. At a measurement location perpendicular to the jet axis, the result was mixed and often an increase in the noise was noted. The latter increase, occurring at large values of $p_{\mu} d / p_{j} D$, was found to be associated with a high-frequency 'cross-over' in the sound pressure level spectra; this occurred with gaseous injection but not with water injection. An interested reader may find further details in the cited reference.

The study was continued to explore the effect of the $\mu$ jets on supersonic jet noise. Three convergent-divergent nozzles, having the same exit diameter and outer geometry as the convergent nozzle used in the earlier study, were fabricated for this purpose. The combination of nozzles allowed an exploration of the $\mu$ jet effect in various flow regimes. It is well known that supersonic jets, when run at off-design condition, produce shock-associated noise in addition to turbulent mixing noise. Two such shock-associated noise components have been studied widely: screech tones $[5,6]$ and broadband shock-associated noise (BBSN) $[6,7]$. These two components trace to interactions between the turbulent flow structures and the shock-train that form downstream of the nozzle in the jet plume. Relatively less known are the noise components that occur when the shock resides within the divergent section of the nozzle. With that flow condition, there can be a resonance yielding 'transonic tones' [8]. Even in the absence of any tones such jets are found to involve an elevated level of broadband noise, termed 'excess broadband noise' (EBBN), as reported in [9].

In the present study, effects of $\mu$ jet injection on the various noise components have been explored covering different flow regimes. The objective of this paper is to summarize the results 
obtained so far. Effects of the injection on overexpanded and underexpanded conditions as well as one case of fully expanded condition are described. The effect of the injection on fully expanded condition is compared with earlier subsonic results and an attempt is made to understand how the individual noise components are affected.

\section{Experimental Procedure}

The experiments were conducted in an open jet facility at NASA Glenn Research Center. Three convergent-divergent (C-D) nozzles and a convergent nozzle were used, all having the same exit diameter of $37.6 \mathrm{~mm}$. Each of the four nozzles was $10 \mathrm{~cm}$ long and could be screwed to a fixed receptacle. The receptacle was attached to the $76.2 \mathrm{~cm}$ diameter plenum chamber of the jet facility (Fig. 1a). The internal diameters and the slopes at the junction between the screw-in piece (marked ' $B$ ' in Fig. 1a) and the receptacle (marked ' $A$ ') were matched so that the two together formed the full nozzle, contracting from $13.1 \mathrm{~cm}$ inlet diameter to the dimensions of the given nozzle. The divergent sections of the nozzles were designed following the method of characteristics. The length of the divergent section varied depending on the design Mach number; thus, the length of the convergent section also varied. A fourth order polynomial fit was used to design the convergent section, matching the slope of the upstream receptacle $(-0.32)$ and bringing the slope to zero at the throat. The interior contours of the four nozzles are shown in Fig. 1(b) and the essential dimensions are listed in Table 1.

\begin{tabular}{|c|c|c|c|c|c|}
\hline Nozzle & $M_{D}$ & $\begin{array}{c}\text { Throat } \\
\text { diameter, } D_{t} \\
(\mathrm{~cm})\end{array}$ & $\begin{array}{c}\text { Divergent } \\
\text { section } \\
\text { length }(\mathrm{cm})\end{array}$ & $\begin{array}{c}M_{j}- \\
\text { sub }\end{array}$ & $\begin{array}{c}M_{j}- \\
\text { shock }\end{array}$ \\
\hline M10 & 1.0 & 3.759 & 0 & -- & -- \\
\hline M15 & 1.5 & 3.466 & 3.981 & 0.610 & 0.779 \\
\hline M18 & 1.8 & 3.139 & 4.943 & 0.454 & 0.842 \\
\hline M22 & 2.2 & 2.659 & 6.298 & 0.305 & 1.026 \\
\hline
\end{tabular}

Table 1 Nozzle dimensions and other characteristics; all nozzles (screw-in pieces) are $10 \mathrm{~cm}$ long and have exit diameter $D=3.76 \mathrm{~cm}$.

The 'fully-expanded jet Mach number,' $M_{j}=\left(\left(\left(p_{j 0} / p_{a}\right)^{(k-1) / k}-1\right) \frac{2}{k-1}\right)^{1 / 2}$, is used as independent variable in the study. Note that it is a fictitious Mach number at off-design run conditions in the supersonic regime. It represents the Mach number had the flow expanded fully for a given pressure ratio, $p_{j 0} / p_{a}$, where $p_{j 0}$ and $p_{a}$ are the jet plenum and ambient pressures, respectively. In Table $1, M_{j}$-sub and $M_{j}$-shock represent values of $M_{j}$ when the shock is at the throat and at the exit, per one-dimensional nozzle flow analysis. The notation $M_{D}$ represents the 
design Mach number; nozzles with $M_{D}=1.0,1.5,1.8$ and 2.2 are designated as M10, M15, M18 and M22, respectively. (Note that the same notations were used in [9] but for a different set of nozzles).

The $\mu$ jets were injected via a ring manifold (marked ' $C$ ' in Fig. 1a). The outer geometry of all nozzles was the same so that, when mounted and pressed all the way in, the manifold sat flush with the nozzle exit. There were six threaded outlets on the manifold that could be fitted with tiny injectors. For the present study, six cylindrical injectors each with diameter $d=0.203 \mathrm{~mm}$ were used. The injection was perpendicular to the primary jet axis. A close-up view of the manifold, with one of the injectors in the foreground, is shown in Fig. 1(c); further description of the manifold and the injectors can be found in [4]. The injection fluid was air from a pressurized source ('K-bottle') and all experiments were conducted for cold flow, i.e., the total temperature of the jet and the $\mu$ jet was the same as in the ambient.

The exit flow properties of the $\mu$ jets including the mass fraction were calculated from the supply pressure $p_{\mu}$ and the port diameter, using isentropic nozzle flow equations [4]. Far-field noise was measured with (B\&K model 4135) microphones located at $\theta=90^{\circ}, 60^{\circ}$ and $25^{\circ}$; the polar location $\theta$ was referenced with respect to the downstream jet axis. The distances of the three microphones from the nozzle exit were 33, 34.9 and 57.5 diameters, respectively. Most of the data presented are for $\theta=90^{\circ}$ and $25^{\circ}$. The spectral data were acquired by a PC based system coupled to National Instruments hardware using Labview software. Spectral analysis was done digitally and the OASPL data were obtained by integration of the spectra. All data acquisition, activation of the $\mu$ jet as well as setting of regulator pressure were done under remote computer control.

Noise source locations, and the effect of $\mu$ jets thereupon, were investigated briefly by the phased-array technique [12]. The data acquisition system consisted of 48 microphones flushmounted to a $1 \mathrm{~m} \times 1 \mathrm{~m}$ aluminum plate. The microphones were arranged in a series of 'log spirals.' The data were reduced using classical 'beamforming' in the frequency domain that assumed stationary, incoherent, point sources. The measured acoustic source region was superimposed on the top of an image of the flow-field taken by a camera placed near the center of the microphone array. The output was a stack of beamform maps, each corresponded to a different frequency band and showed the location of the dominant noise source or sources for that band, as 2D color contours. The dynamic range of the contours (maximum minus minimum value) was the same for each map but the peak value varied from map to map. An interested reader may find further details of the technique in the cited reference. 


\section{Results}

Noise characteristics of the four nozzles without any $\mu$ jet injection are explored first. The results are shown in Figs. 2(a)-(d). Overall sound pressure levels, measured at the three polar locations, are plotted as a function of $M_{j}$. The vertical lines in these figures demarcate flow regimes based on one-dimensional nozzle flow analysis (long-dashed line: when throat is choked; short-dashed line: when the shock exits the nozzle; chain-dashed line: when flow is fully-expanded). In each figure, also shown are the tone frequency data as the pressure ratio (and hence, $M_{j}$ ) is varied; the scale for the frequency is indicated on the top right of each figure. The frequency data represent screech tones [5] as well as transonic tones (' $x$-tones') occurring with the C-D nozzles [8], as marked in the figures. For the convergent case in Fig. 2(a) the wellknown screech stages $\left(A_{1}, A_{2}, B, C, D\right.$ and $\left.E\right)$ are observed and these are identified in the caption. With the M15 nozzle (in Fig. 2b), only three stages of screech were observed in the $M_{j}$ range 1.1 - 1.8. By comparison with the convergent case, these appear to be (from left to right) the axisymmetric (A), flapping (B) and again another flapping mode (D). Corresponding data for the M18 and M22 cases are shown in Figs. 2(c) and (d), respectively. With each, loud transonic tones were observed in the range $M_{j}<1.1$. Each of them also involved screech at high $M_{j}(>1.4)$.

When the tones occurred, naturally the overall sound pressure level was also relatively high. This partially caused the bumps and undulations in the OASPL data. For example, a mild bump is noted in Fig. 2(a) for the convergent case around $M_{j}=1$.3. This corresponds to the flapping (B) mode screech. A similar bump is noted in Fig. 2(b) around the same $M_{j}$ location. Among the different screech modes, the flapping mode appears to yield the largest increase in the OASPL. It has been shown before that this mode also causes a large increase in the jet spreading rate $[10,11]$. The transonic tones also result in large increases in the OASPL causing bumps in the curves in the range $0.6<M_{j}<1$; see Figs. 2(c) and 2(d). It is emphasized that the increases in the OASPL and the resulting bumps are not only due to screech and $x$-tones. Broadband noise components BBSN and EBBN also contribute. For example, the bump in Fig. 2(b) around $M_{j}=0.85$ is attributable mainly to EBBN since x-tones were absent; the contributions of EBBN and BBSN are discussed further in the following.

In Fig. 2(b), it is also noted that there is a conspicuous dip in the curves at $M_{j}=1.5-$ especially at the larger $\theta$ locations. This is because the flow is fully expanded at this $M_{j}$ for the M15 nozzle and the noise is due only to turbulent mixing. A similar trend is seen for the M18 case (Fig. 2c) where the amplitude drops as $M_{j}=1.8$ is approached. This attests to the fact that the nozzles have been designed well and nearly shock-free operation is achieved at the design 
condition. Because of supply pressure limitation with the facility, the fully expanded condition could be obtained only with the M15 nozzle and not with the nozzles with higher $M_{D}$.

The same OASPL data from Fig. 2 are replotted to directly compare the trends for the four nozzles. These are shown in Figs. 3(a) and 3(b) for $\theta=25^{\circ}$ and $90^{\circ}$, respectively. It can be seen that the C-D nozzles are significantly louder than the convergent nozzle in the low end of the overexpanded regime (approximately for $M_{j}<1.2$ ). The increase in the noise is attributable only partly to the x-tones. In fact, the increased levels are primarily due to the broadband component EBBN. The EBBN, noticeable in the range of $0.3<M_{j}<1.2$, becomes more pronounced with nozzles of higher design Mach number; an interested reader may find further details of these characteristics in [9].

The effect of $\mu$ jet injection on the sound pressure level spectra is now considered. Figure 4 shows spectral data with and without $\mu$ jet injection, for the convergent nozzle. Data for three values of $M_{j}$ are shown in Figs. 4(a), (b) and (c), as examples. At each $M_{j}$, data for injection at $p_{\mu}$ $=6200 \mathrm{kPa}$ (dashed curves) are compared to the no-injection case (solid curves). The pair of curves at the top of each figure are for $\theta=25^{\circ}$ (with scale on left) and the pair at the bottom are for $\theta=90^{\circ}$ (with scale on right). For the subsonic case in Fig. 4(a), it can be seen that $\mu$ jet injection has reduced the energy near the low-frequency peak (turbulent mixing noise) but at the expense of an increase at high frequencies. The OASPL (shown at the last column of the legend) has dropped by about $1.5 \mathrm{~dB}$ at $\theta=25^{\circ}$ but increased by about $1 \mathrm{~dB}$ at $\theta=90^{\circ}$. The parametric dependence of OASPL reduction for subsonic jets and phenomena such as the highfrequency cross-over of spectral energy have been discussed in [4] and these will not be repeated here. Turning attention to the supersonic condition, it can be seen in Fig. 4(b) that screech (mode $\mathrm{B} ; M_{j}=1.27$ ) has been eliminated by the injection. In addition, broadband levels have also decreased. Broadband shock-associated noise (BBSN), characterized by the hump on the right in the $90^{\circ}$ spectra, apparently has been affected little. In fact, with injection it has been 'unearthed' in this case since the high levels due to screech have been reduced. At the highest $M_{j}$ in Fig. 2(c), similar but less pronounced effects of the injection are observed.

Examples of spectral data for the M15 nozzle are similarly shown in Fig. 5. Trends similar to those noted with Fig. 4 are observed. However, although the broadband energy has been reduced screech has not been eliminated completely (Fig. 5a). At $M_{j}=1.511$ (only slightly off from fully-expanded condition, Fig. 5b), the $\mu$ jet effect on the OASPL is relatively small but still substantial. There is about $1.5 \mathrm{~dB}$ reduction in OASPL at $25^{\circ}$ but an increase at $90^{\circ}$. At the highest $M_{j}$ (Fig. 5c) the effect is again less pronounced. 
Corresponding spectral data for the M18 and M22 nozzles are shown in Figs. 6 and 7, respectively. It can be seen that with the M18 case, in Fig. 6(a), the x-tone has persisted under the injection although both amplitude and frequency have decreased somewhat. At lower values of $M_{j}$ with this nozzle ( 0.6), the x-tone could be completely eliminated by the injection; this will be evident from the OASPL reduction plots shown in the following. With the M22 case, on the other hand, the injection was ineffective in eliminating the $\mathrm{x}$-tones at any value of $M_{j}$; examples can be seen in Fig. 7(a) and (b). Screech tone was also affected little for this nozzle (Fig. 7c). Thus, while screech is eliminated by the injection with the convergent nozzle, the effect progressively diminishes with nozzles of higher design Mach number. Similarly, the effect on transonic tone also diminishes with nozzles of higher design Mach number. For the higher $M_{D}$ nozzle, since the angle of divergence is greater the diminishing effect of the $\mu$ jet is likely to be due to the fact that the injection location is farther away from the core of the jet. The problem is aggravated by boundary layer separation which is expected to occur downstream of the shock, with the higher $M_{D}$ nozzles.

The reductions in the OASPL due to $\mu$ jet injection for the convergent nozzle are shown in Fig. 8 (a) and (b) for $\theta=25^{\circ}$ and $90^{\circ}$, respectively. The solid curves are generated by polynomial fits through the data. The data has considerable scatter and too much emphasis must not be placed on the exact trends exhibited by these curves. In fact, when the scatter is too large (e.g. at $M_{j}=1.276$ ) the fitted curves show trends that may not be meaningful and thus are not shown. The data are plotted as a function of the normalized pressure ratio; this is the parameter that was found to correlate the OASPL reduction data at shallow angles for subsonic jets [4]. As stated before, the subsonic correlation applies for only shallow angular locations and it is shown by the dotted line in Fig. 8(a). It can be seen that reductions as large as $5 \mathrm{~dB}$, much more than that observed at the subsonic condition, are achieved with the supersonic jets. The reductions are only partly due to the elimination of screech. Referring back to the SPL spectra in Fig. 4(b) it can be seen that most of the reduction yields from a decrease in the broadband levels. At $M_{j}=1.276$ for $\theta=90^{\circ}$, as high as $7 \mathrm{~dB}$ reduction in OASPL is obtained due to elimination of B-mode screech and the associated broadband levels. For the latter angular location, some of the cases (e.g., at $M_{j}=1.471$ ) exhibit an increase in the OASPL (negative ordinate).

Corresponding data for the M15 nozzle are shown in Fig. 9. For $M_{j}=1.516$ which is only slightly off from the design condition, the OASPL reduction at $\theta=25^{\circ}$ is significantly larger than the subsonic correlation (Fig. 9a, triangular data points). However, the reduction becomes less as the design condition is closely matched, as discussed shortly. For the M18 case (Fig. 10), OASPL reductions as large as $10 \mathrm{~dB}$ are achieved at $M_{j}=0.64$ when the x-tone is completely 
eliminated by $\mu$ jet injection. The large effect is also due to a reduction in EBBN. Figure 11 shows the OASPL reduction data for the M22 case representing the nozzle with highest design Mach number tested. The overall effect of the $\mu$ jets is relatively less (within $2 \mathrm{~dB}$ at $\theta=25^{\circ}$ ) - although the effect is still comparable or better than the subsonic correlation.

Figure 12 shows another set of OASPL reduction data closer to the design condition for the M15 nozzle $\left(M_{j}=1.501\right)$. For clarity this data-set is shown separately and not with corresponding data in Fig. 9(a); the set of data for $M_{j}=1.516$ has been repeated for clear comparison. The OASPL reductions now fall closer to the subsonic correlation. There is deviation at higher $\mu$ jet pressures (by about $0.5 \mathrm{~dB}$; note that ordinate scale is expanded relative to that in Fig. 9a). However, a completely shock-free and perfectly expanded supersonic jet is hard to achieve and the deviation is probably due to residual shock effect. The deviation is also approximately within the data scatter range in [4]. Thus, it appears that the subsonic correlation (relevant to turbulent mixing noise reduction) applies to fully-expanded supersonic jets too. Perhaps, this is not a surprise since a fully-expanded jet only involves turbulent mixing noise. For jets at off-design run conditions, on the other hand, the injection additionally reduces shockassociated noise. Thus, more pronounced noise reduction is achieved with the latter jets; this is underscored by the data in Fig. 12.

Thus, among the four nozzles the greatest noise reduction is noted with the M18 case. The overall $\mu$ jet effect for this nozzle is shown in Fig. 13 for injection pressure $p_{\mu}=6200 \mathrm{kPa}$. For reference, corresponding spectral data can be found in Fig. 6 . The injection has reduced the levels substantially -- by more than $10 \mathrm{~dB}$ in the range, $0.5<M_{j}<0.75$. The jet corresponding to several of the data points within this range do not involve $x$-tones and the reduction is due only to an effect on the broadband component EBBN. It is also noted that at no condition, in the $M_{j}$ range of $0.5-1.2$, do the OASPL levels drop to coincide with the convergent case levels. That is, EBBN could not be completely eliminated by the injection within the parameters covered. However, there is clearly a favorable effect of the injection towards reducing EBBN. This is further illustrated in Fig. 14 for two cases; (a) M15 nozzle at $M_{j}=1.02$ and (b) M18 nozzle at $M_{j}$ $=1.16$. For each case and at each angular location there is a general reduction in the broadband levels. In Fig. 14(a), a 'cross-over' in the amplitudes is noted for $\mathrm{f}>20 \mathrm{kHz}$. This appears to be mostly due to noise from the $\mu$ jets themselves. The cross-over is absent in Fig. 14(b) because the baseline noise is already high - more than $5 \mathrm{~dB}$ larger than that for the M15 case in Fig. 14(a), rendering the noise from the $\mu$ jets less significant. As stated earlier, further discussion on the 'cross-over' and $\mu$ jet self-noise can be found in [4]. 
With regards to the effect on BBSN, it appears that $\mu$ jets in most cases aggravate the situation. We have seen in Figs. 4 and 5 that BBSN was 'unearthed' by the injection as screech noise was reduced. Figure 15 documents two additional cases, for the M10 and M15 nozzles, where BBSN has been accentuated by the injection. This can be seen from the $90^{\circ}$ spectra in either figure. For the M15 case (Fig. 15b), a relatively higher injection pressure (10.3 MPa, $p_{\mu} d / p_{j} D \approx 0.13$ ) has been used to delineate the point. While about $2.3 \mathrm{~dB}$ reduction in OASPL has occurred at $25^{\circ}$, an increase by about $3.5 \mathrm{~dB}$ has occurred at $90^{\circ}$. The latter increase is due mostly to an accentuation of BBSN as clear from spectra in Fig. 15(b).

Finally, effects of $\mu$ jet injection on noise source location are briefly discussed. A set of data for the baseline flow (without injection) is first presented in Fig. 16. These data are for the M22 nozzle at $M_{j}=1.042$, involving a 'dull' $x$-tone at $2.236 \mathrm{kHz}$. Noise spectrum, taken simultaneously with the phased-array data, by one of the microphones in the array is shown at the top (corresponding far-field SPL spectra are in Fig. 7b). As stated in the experimental procedure section, the output of the phased-array measurement was a stack of contours at 84 frequencies over the spectrum. The contours at only 5 frequencies $(0.63,2,2.236,10$ and 25 $\mathrm{kHz}$ ) are shown in Fig. 16, as examples; the frequency locations are marked in the spectrum. The brightest regions in these plots denote the source of most intense noise radiation at corresponding frequencies. One finds that at all frequencies, including at the $\mathrm{x}$-tone, the sources are centered right at the nozzle exit. It should be noted that the same observation was made either for sharp $\mathrm{x}$-tones or when the tone was not visible in the spectrum and the noise was due only to EBBN. This provided a confirmation of earlier inference $[8,9]$ that these two components are due to unsteady shock motion within the divergent section of the nozzle.

With injection, yielding significant noise reduction, no clear change in the extent and the location of the source could be detected. Two sets of data showed a curious effect of the injection and these are included in Fig. 17 for a high subsonic case and in Fig. 18 for a supersonic case. In each figure, four frames are shown for four frequency bands as indicated. In each frame, the upper picture is for the baseline flow without any injection while the lower picture is with injection. In the subsonic case at $M_{j}=0.95$ (Fig. 17), the noise source is drawn closer to the nozzle under the injection at all frequencies (e.g., from about $5 \mathrm{D}$ to $2 \mathrm{D}$ at $10 \mathrm{kHz}$ ). At the supersonic condition at $M_{j}=1.27$ (involving B-mode screech, Fig. 18), on the other hand, the effect is opposite. The source at all frequencies has elongated and been pushed farther downstream by the injection (e.g., from 8D to 10D at $10 \mathrm{kHz}$ ). At the highest frequency (Fig. 18), the shock train has apparently shrunk but the source region has elongated under the injection. $A$ satisfactory explanation for the observed effects seen with the phased-array data, and for the 
underlying mechanism of noise suppression, could not be reached at this time and will require further investigation.

\section{Conclusions}

The effect of microjet injection has been investigated for supersonic jets covering both underexpanded and overexpanded conditions as well as one fully expanded condition. Relative to the effect on subsonic jets, larger reductions in the overall sound pressure levels are achieved in most supersonic conditions. Very large reductions are typically associated with suppression of screech and transonic tones. This involves not only the elimination of the tones but also a concomitant reduction in broadband levels. For a shock-free fully expanded case, the reductions achieved are comparable to that in the subsonic case; the same correlation found for subsonic jet noise reduction at shallow observation angle also applies. The data also suggest that $\mu$ jet injection has the promise for reduction of the broadband noise component EBBN occurring with overexpanded jets. However, it appears that the component BBSN occurring in both overexpanded and underexpanded regimes may get accentuated under the injection. Thus, for a fully-expanded case while the injection reduces noise in the direction of peak noise radiation an increase is noted in the perpendicular direction due to an elevated level of BBSN.

\section{Acknowledgement}

Support from the Supersonics Project, under the NASA Fundamental Aeronautics Program, is gratefully acknowledged.

\section{References:}

[1] Krothapalli, A., Venkatakrishnan, L., Lourenco, L.M., Greska, B. and Elavarasan, R., "Turbulence and noise suppression of a high-speed jet by water injection," J. Fluid Mech., 491, 2003.

[2] Castelain, T., Sunyach, M., Juve, D. and Bera, J.-C., "Jet-noise reduction by impinging microjets: an acoustic investigation testing microjet parameters," AIAA J., 46(5), May, 2008.

[3] Henderson, B., "Over 50 years of fluidic injection for jet noise reduction", Int. J. of Aeroacoustics (to appear), 2009

[4] Zaman, K.B.M.Q., "Subsonic jet noise reduction by microjets - a parametric study," International J. of Aeroacoustics (to appear). (presented earlier as AIAA Paper 2009-3129, $15^{\text {th }}$ AIAA/CEAS Aeroacoustics Conference, Miami, FL., May 11-13, 2009.)

[5] Powell, A., "On the mechanism of choked jet noise," Proc. Phys. Soc. London, B 66, pp. 1039-1056, 1953.

[6] Tam, C.K.W., "Supersonic jet noise," Ann. Review Fluid Mech., 27, pp. 17-43, 1995.

[7] Harper-Bourne, M. and Fisher, M.J., "The noise from shock waves in supersonic jets," Proc. AGARD Conf. on noise mechanisms (No. 131), Brussels, Belgium, 1973.

[8] Zaman, K.B.M.Q., Dahl, M.D., Bencic, T.J. and Loh, C.Y., "Investigation of a 'transonic resonance' with convergent-divergent nozzles," J. Fluid Mech., 463, pp. 313-343, 2002.

[9] Zaman, K.B.M.Q., Bridges, J.E. and Brown, C.A., "Excess broadband noise observed with overexpanded jets", AIAA J., 48(1), pp. 202-214, 2010.

[10] Zaman, K.B.M.Q., "Spreading characteristics of compressible jets from nozzles of various geometries,"

J. Fluid Mech., 383, pp. 197-228, 1999. 
[11] Glass, D.R., "Effects of acoustic feedback on the spread and decay of supersonic jets", AIAA J., 6, pp. 1890-1897, 1968.

[12] Dougherty, R.P., Podboy, G.G. "Improved phased array imaging of a model jet," AIAA Paper 20093186, 15 ${ }^{\text {th }}$ AIAA/CEAS Aeroacoustics Conference, Miami, FL., May 11-13, 2009. 


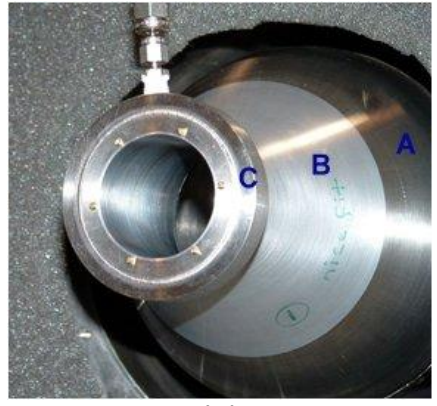

(a)

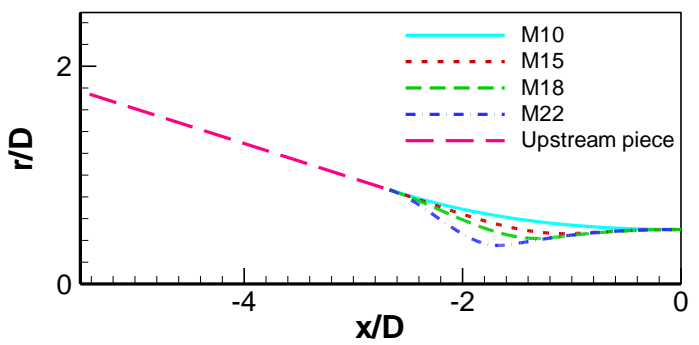

(b)

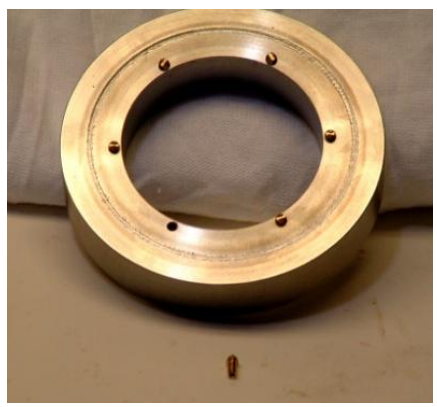

(c)

Fig. 1 Experimental setup; (a) picture of nozzle, (b) nozzle internal contours, (c) $\mu$ jet manifold.

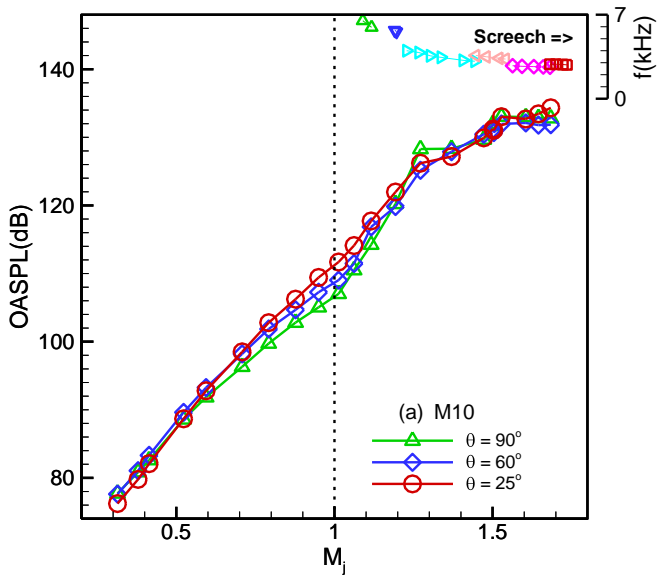

Fig. 2(a) Caption next column.
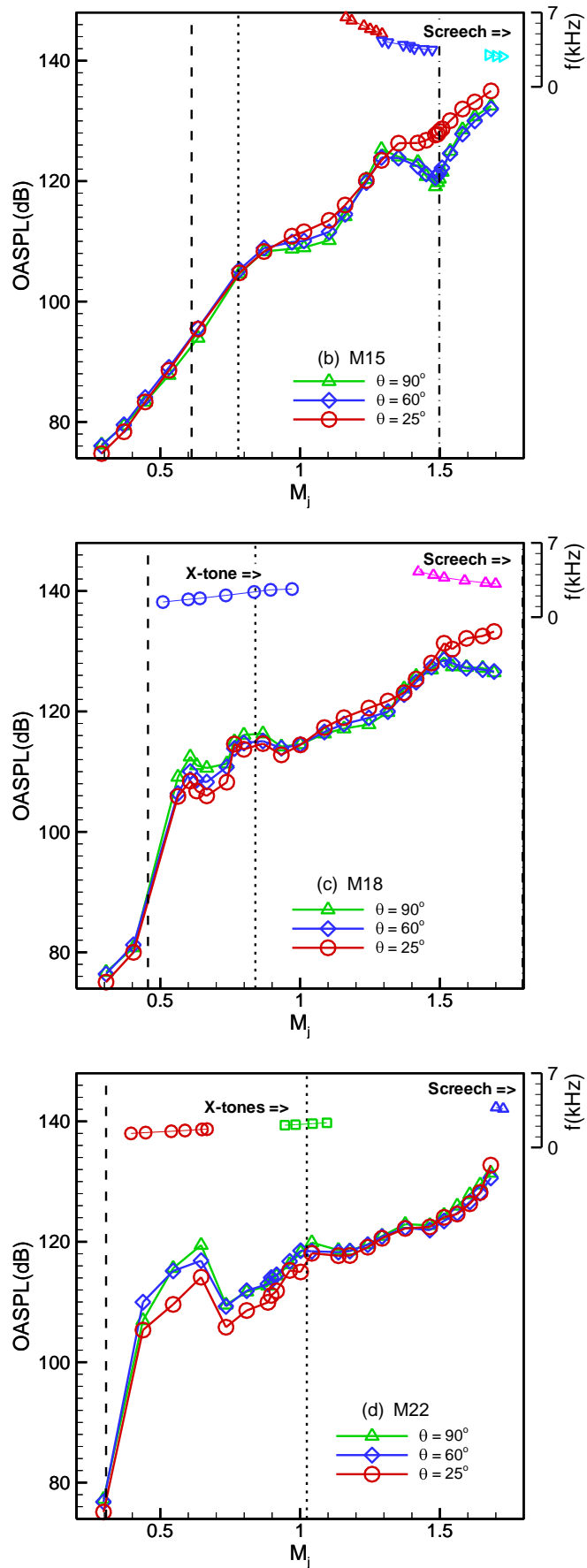

Fig. 2 OASPL versus $M_{j}$ for: (a) M10, (b) M15, (c) M18 and (d) M22 nozzles. In each figure, data are shown for three observation angles. Also shown at the top of each figure are the tone frequency data (scale on top right). Screech modes for the convergent case (Fig. 2a) are: deltas, A1; gradients, A2; right triangles, B; left triangles, C; diamonds, $\mathrm{D}$; squares: $\mathrm{E}$. 

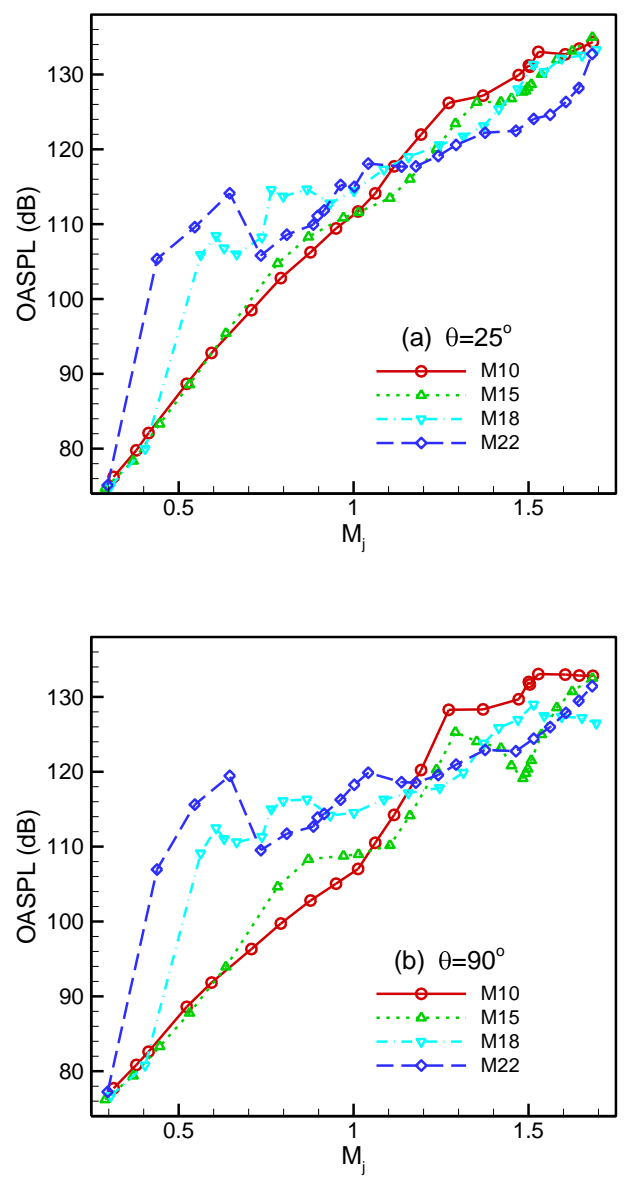

Fig. 3 OASPL versus $M_{j}$ for the four nozzles at: (a) $\theta=25^{\circ}$ and (b) $\theta=90^{\circ}$.
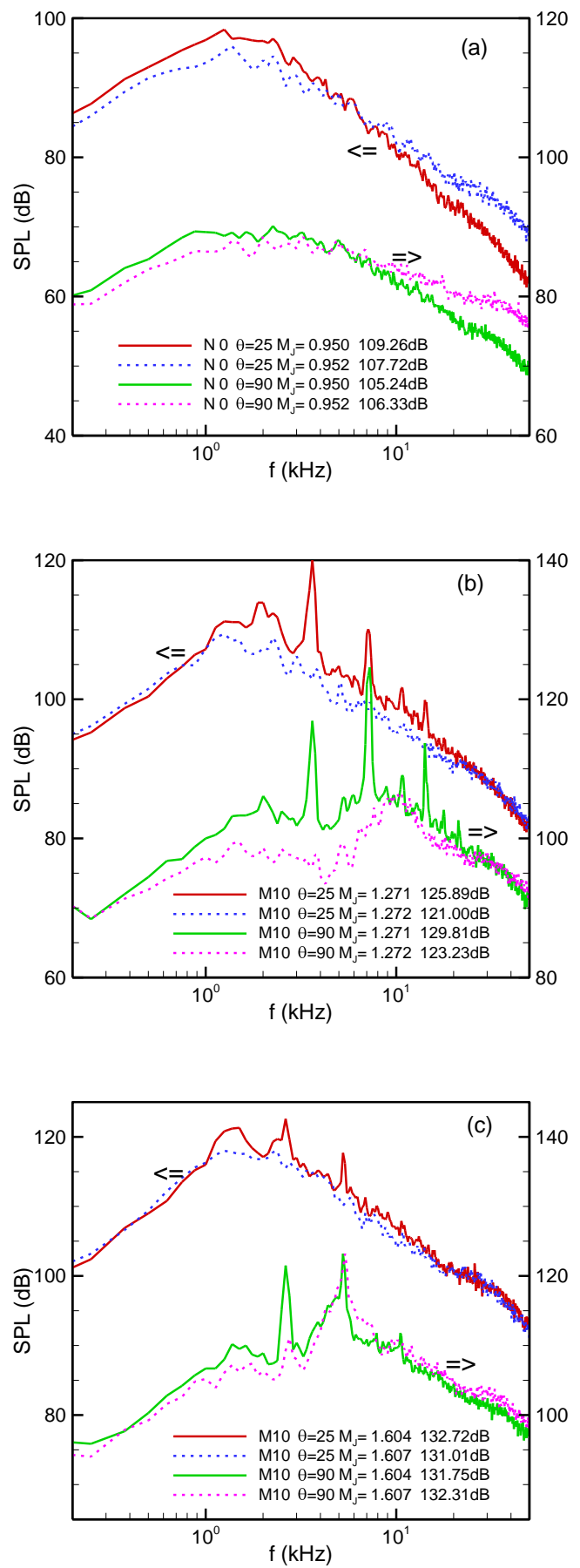

Fig. 4 SPL spectra for the M10 nozzle with and without $\mu$ jet injection at $6200 \mathrm{kPa}$; solid line: no injection, dashed line: with injection. In each figure, data for $\theta=25^{\circ}$ and $90^{\circ}$ are shown, with scale for $25^{\circ}$ on left and for $90^{\circ}$ on right. (a) $M_{j}=$ 0.95, (b) $M_{j}=1.27$ and (c) $M_{j}=1.6$. 

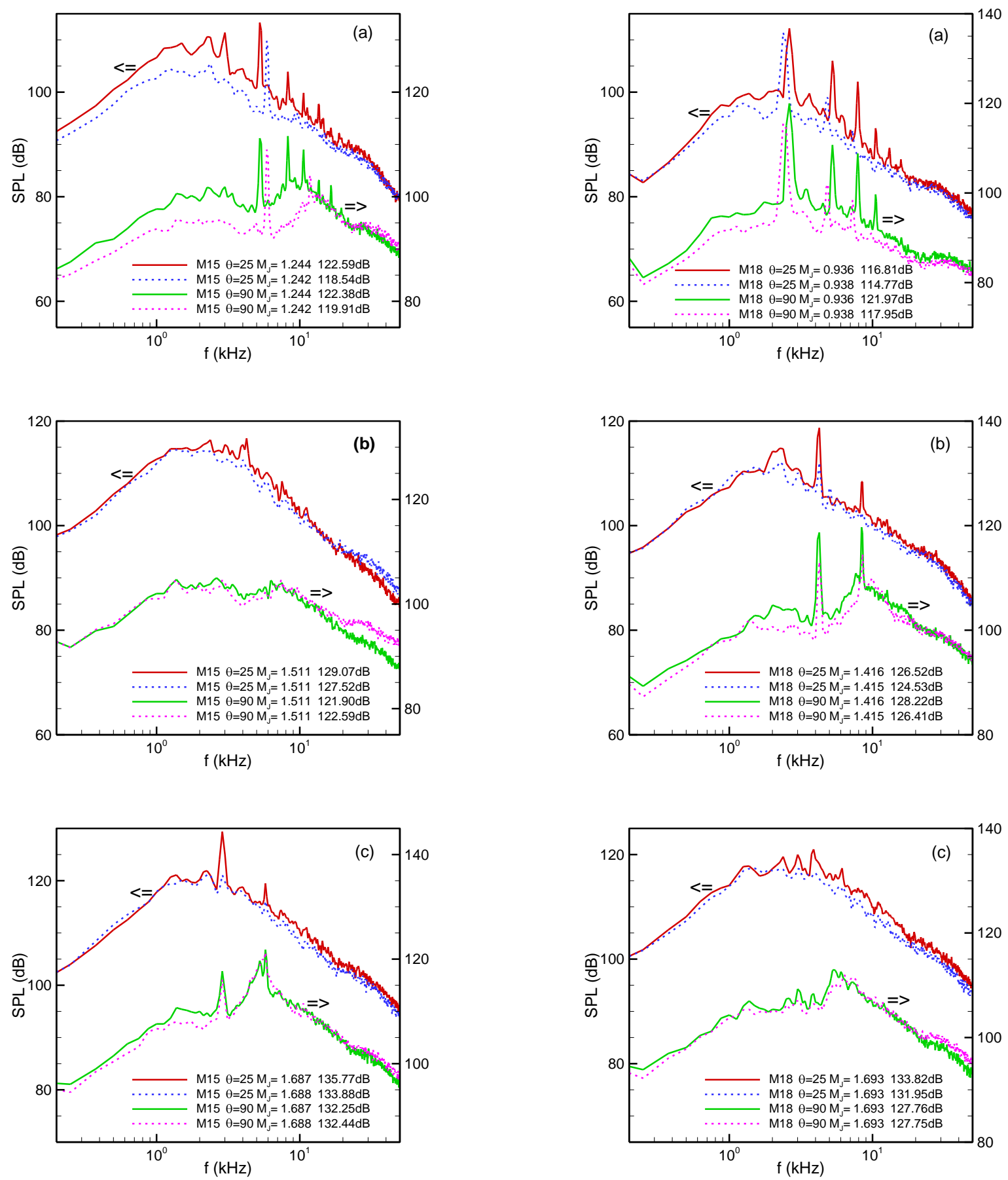

Fig. 5 SPL spectra for the M15 nozzle with and without $\mu$ jet injection at $6200 \mathrm{kPa}$; solid line: no injection, dashed line: with injection. In each figure, data for $\theta=25^{\circ}$ and $90^{\circ}$ are shown, with scale for $25^{\circ}$ on left and for $90^{\circ}$ on right. (a) $M_{j}=$ 1.24, (b) $M_{j}=1.51$ and (c) $M_{j}=1.69$. 

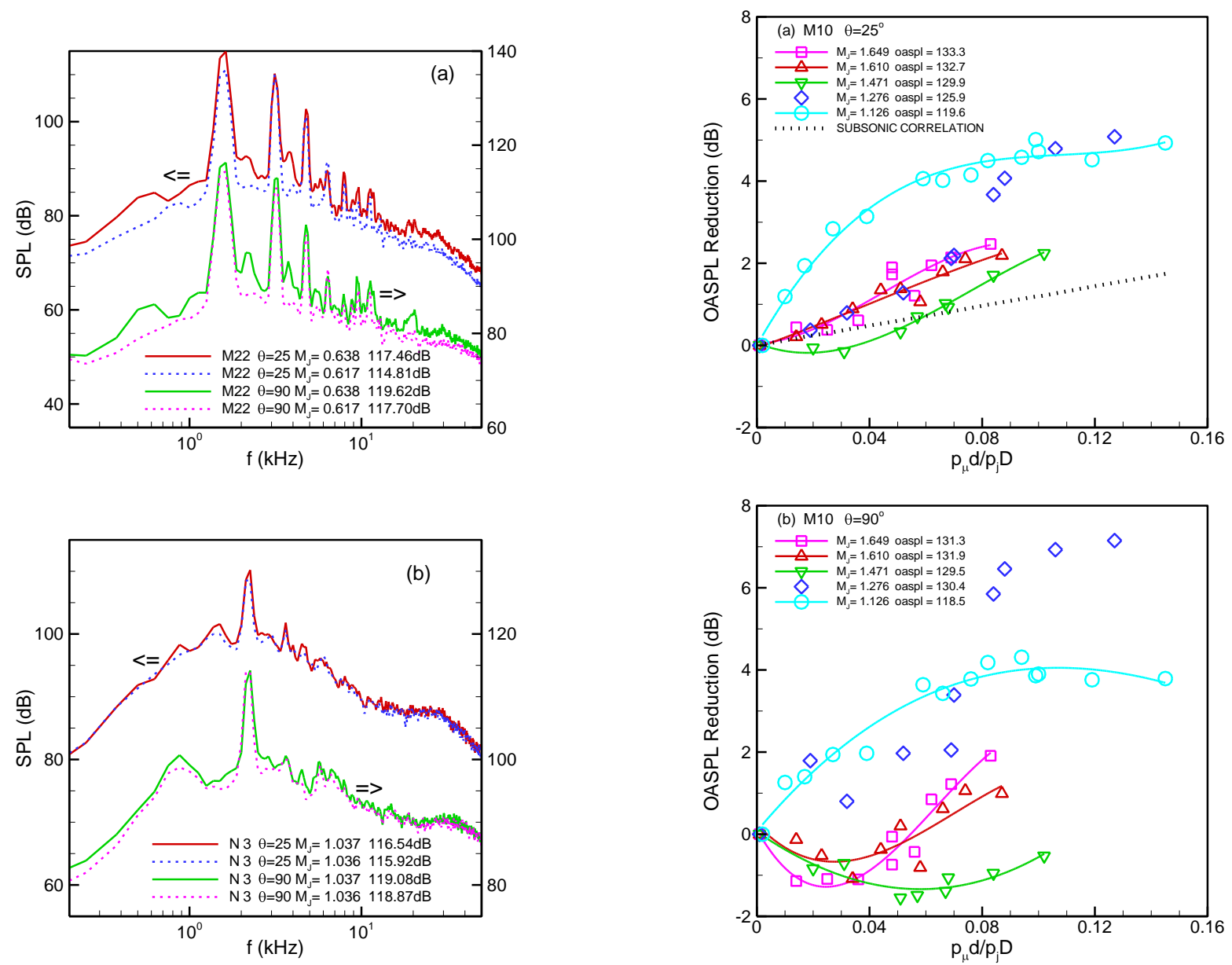

Fig. 8 Reduction in OASPL versus normalized $\mu$ jet pressure for the M10 nozzle. 'Jet Mach number' $M_{j}$ and corresponding baseline OASPL are indicated in legend; (a) $\theta=25^{\circ}$, (b) $\theta=90^{\circ}$.

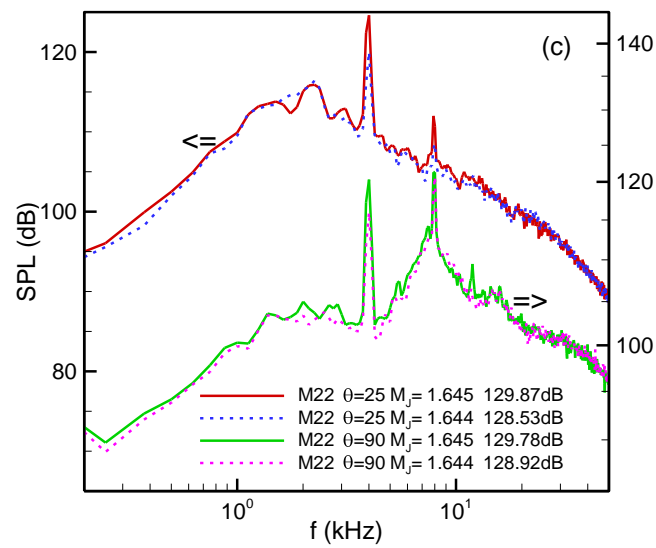

Fig. 7 SPL spectra for the M22 nozzle with and without $\mu$ jet injection at $6200 \mathrm{kPa}$; solid line: no injection, dashed line: with injection. In each figure, data for $\theta=25^{\circ}$ and $90^{\circ}$ are shown, with scale for $25^{\circ}$ on left and for $90^{\circ}$ on right. (a) $M_{j}=$ 0.63, (b) $M_{j}=1.04$ and (c) $M_{j}=1.65$. 

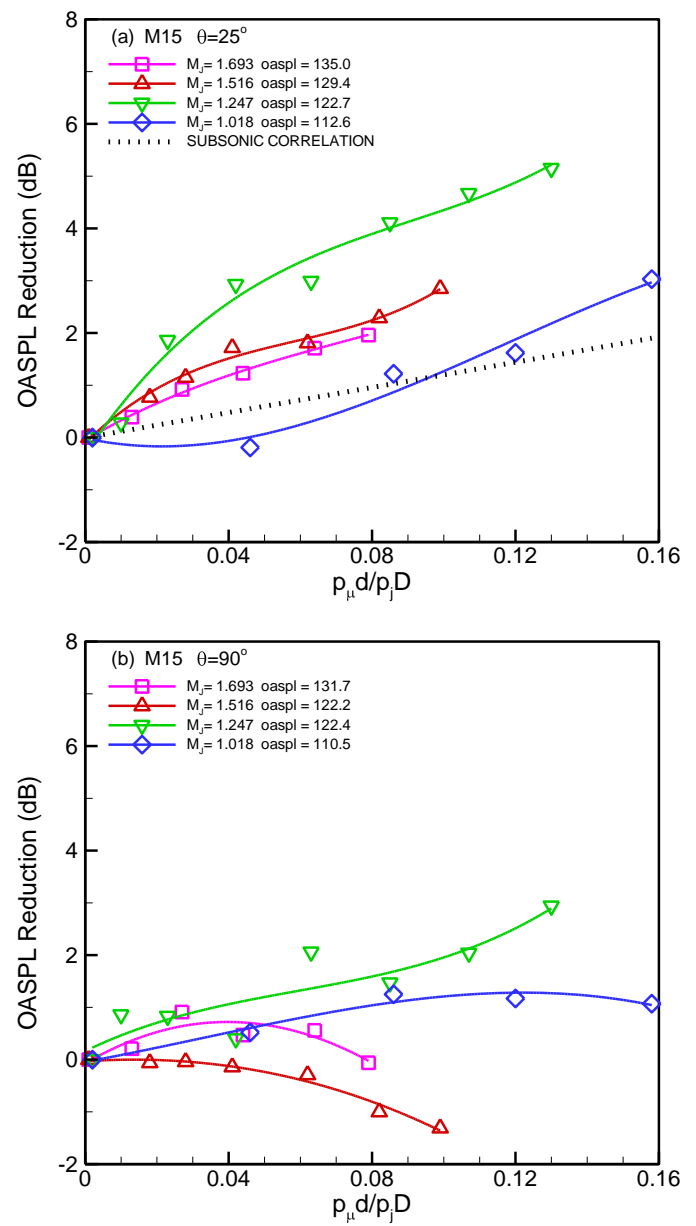

Fig. 9 Reduction in OASPL versus normalized $\mu$ jet pressure for the M15 nozzle. 'Jet Mach number' $M_{j}$ and corresponding baseline OASPL are indicated in legend; (a) $\theta=25^{\circ}$, (b) $\theta=90^{\circ}$.
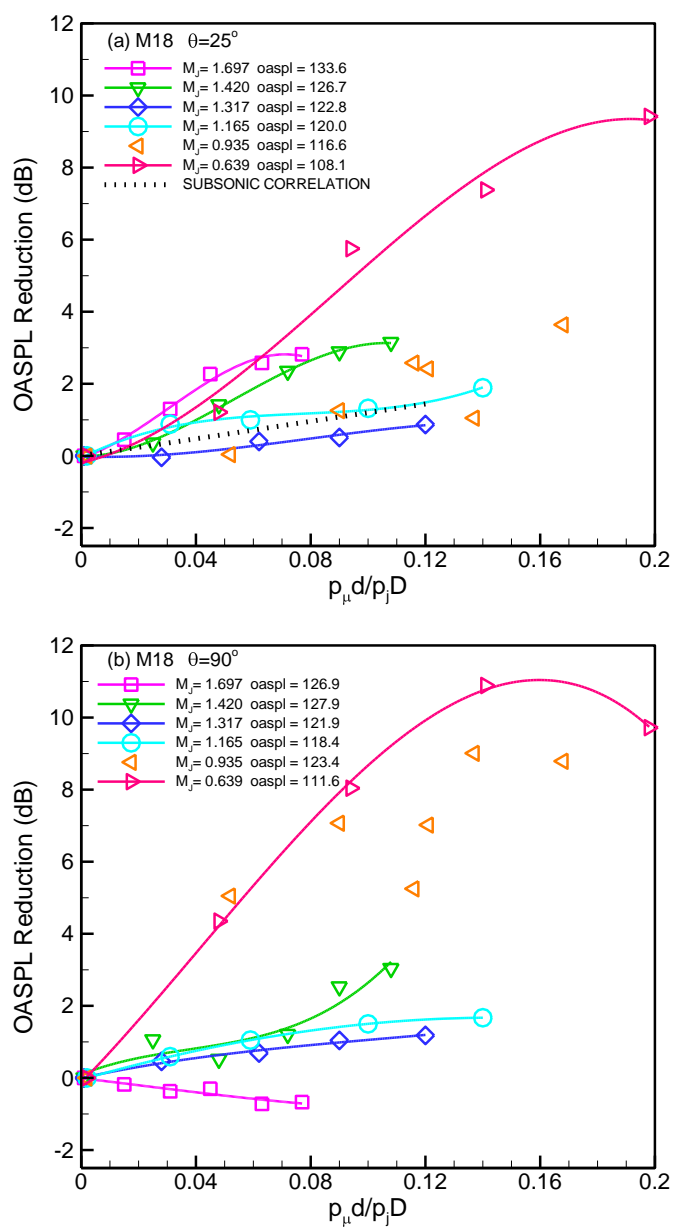

Fig. 10 Reduction in OASPL versus normalized $\mu$ jet pressure for the M18 nozzle. 'Jet Mach number' $M_{j}$ and corresponding baseline OASPL are indicated in legend; (a) $\theta=25^{\circ}$, (b) $\theta=90^{\circ}$. 



Fig. 11 Reduction in OASPL versus normalized $\mu$ jet pressure for the M22 nozzle. 'Jet Mach number' $M_{j}$ and corresponding baseline OASPL are indicated in legend; (a) $\theta=25^{\circ}$, (b) $\theta=90^{\circ}$.

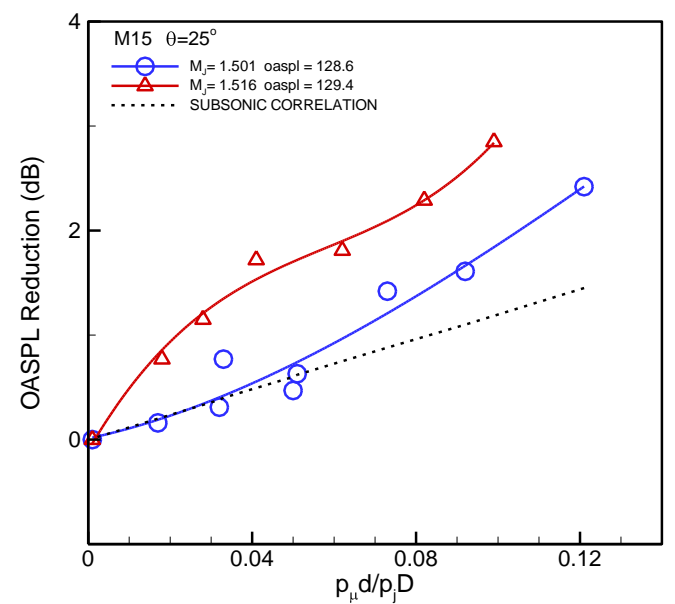

Fig. 12 Reduction in OASPL versus normalized $\mu$ jet pressure for the M15 nozzle, similar to Fig. 9 , with additional set of data at fully-expanded condition. 

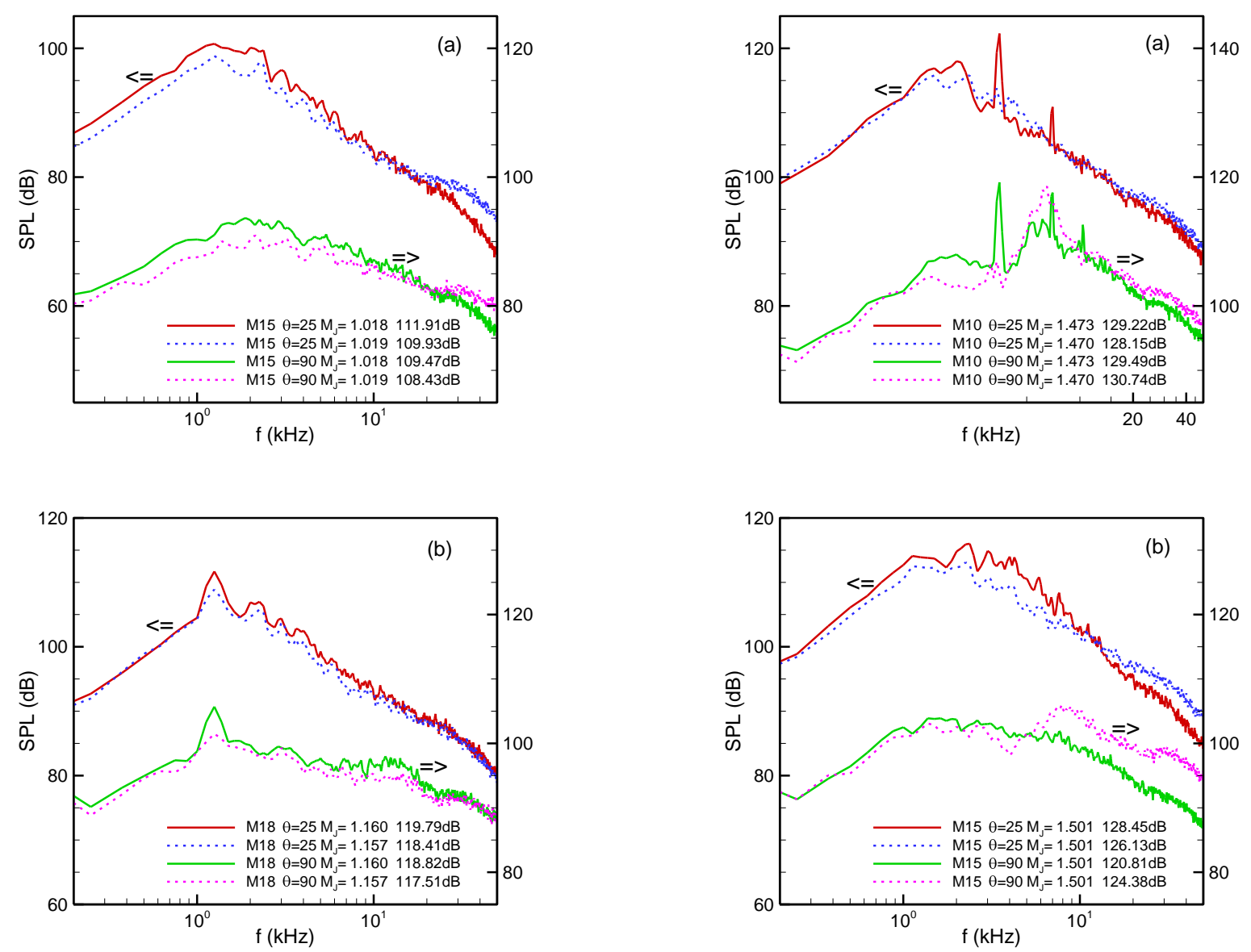

Fig. 14 SPL spectra showing a favorable effect of $\mu$ jet injection on EBBN; solid line: no injection, dashed line: with injection. In each figure, data for $\theta=25^{\circ}$ and $90^{\circ}$ are shown, with scale for $25^{\circ}$ on left and for $90^{\circ}$ on right. (a) M15 case, $M_{j}=1.02,6200 \mathrm{kPa}$, (b) M18 case, $M_{i}=1.16, p_{\mu}=6200 \mathrm{kPa}$.

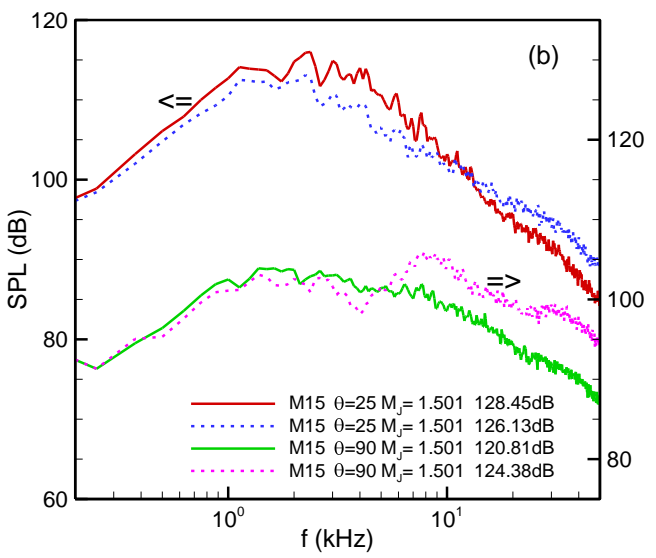

Fig. 15 SPL spectra showing an adverse effect of $\mu$ jet injection on BBSN; solid line: no injection, dashed line: with injection. In each figure, data for $\theta=25^{\circ}$ and $90^{\circ}$ are shown, with scale for $25^{\circ}$ on left and for $90^{\circ}$ on right. (a) M10 case, $M_{j}=1.473,6200 \mathrm{kPa}$, (b) M15 case, $M_{i}=1.501, p_{\mu}=10.3 \mathrm{MPa}$. 


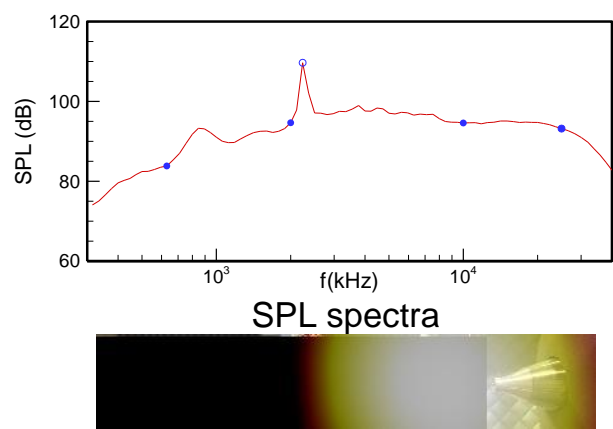

$f=0.63$

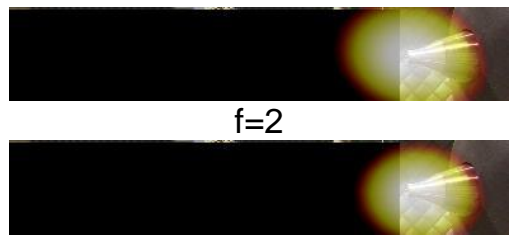

$f=2.236$

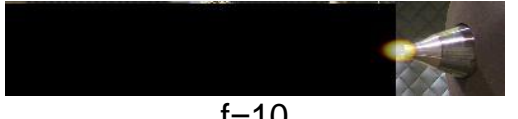

$f=10$

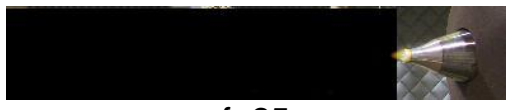

$f=25$

Fig. 16 Noise source location measured by phased-array technique at indicated frequencies $(\mathrm{kHz})$; the frequency locations are marked in the microphone spectra shown at top. Data are for M22 nozzle (no $\mu$ jet injection) at $M_{j}=1.042$ yielding $x$-tone at $2.236 \mathrm{kHz}$.

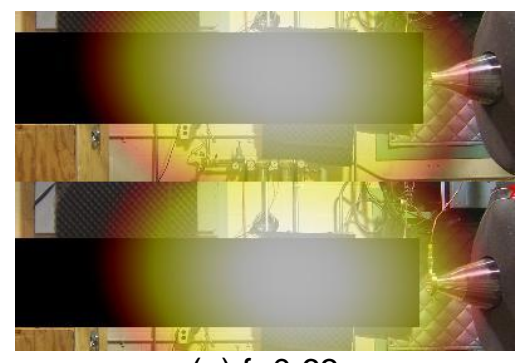

(a) $f=0.63$

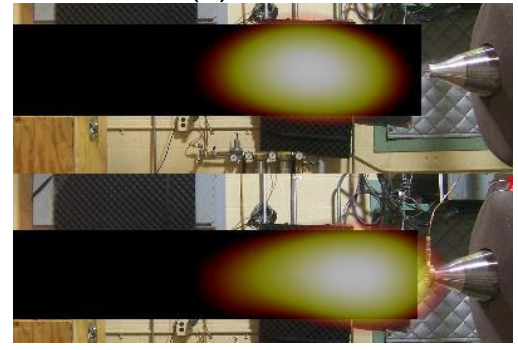

(b) $f=2$

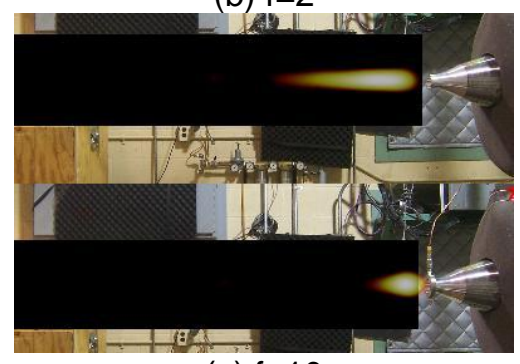

(c) $f=10$

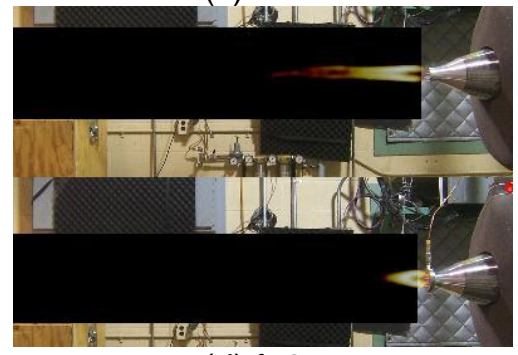

(d) $f=25$

Fig. 17 Noise source location measured by phased-array technique at indicated frequencies $(\mathrm{kHz})$; data are for M10 nozzle at $M_{j}=0.95$. In each frame top picture is for no injection and bottom picture is for $\mu$ jet injection at $6200 \mathrm{kPa}$. 


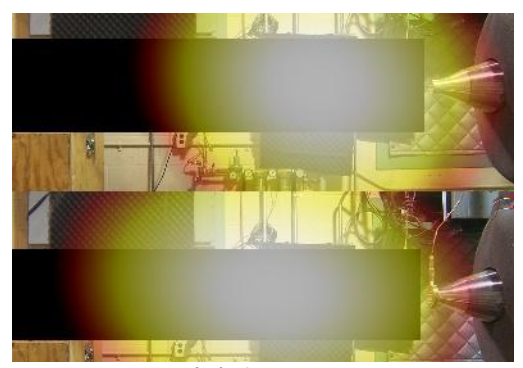

(a) $f=0.63$

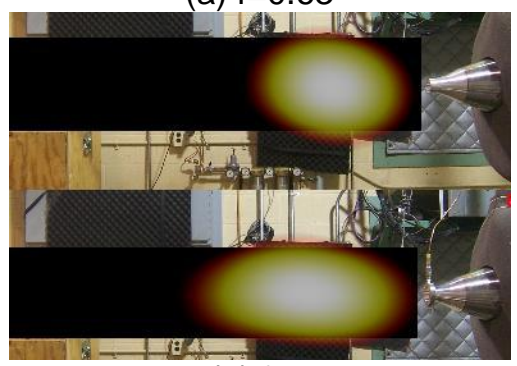

(b) $f=2$

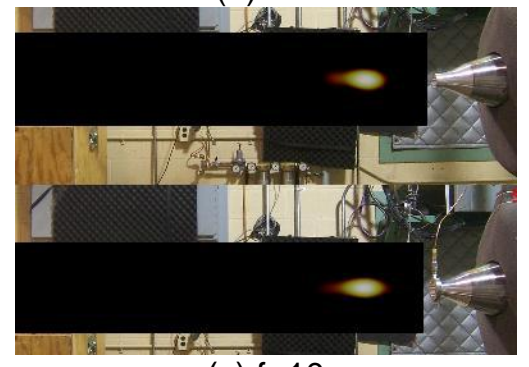

(c) $f=10$

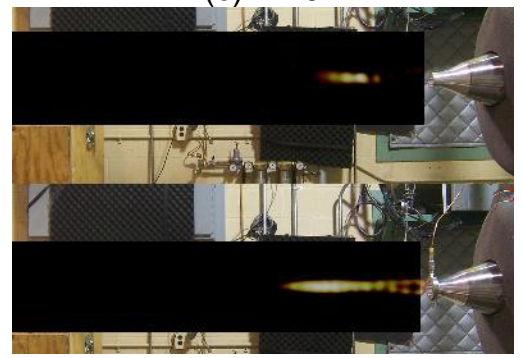

(d) $f=25$

Fig. 18 Noise source location measured by phased-array technique at indicated frequencies $(\mathrm{kHz})$; data are for $\mathrm{M} 10$ nozzle at $M_{j}=1.27$ involving B-mode screech. In each frame top picture is for no injection and bottom picture is for $\mu$ jet injection at $6200 \mathrm{kPa}$. 